\title{
Healthcare System Technology using Smart Phones and Web Apps (Case Study Iraqi Environment)
}

\author{
Suhiar Mohammed Zeki ${ }^{a}$, Dr.Abdul Monem Saleh Rahma ${ }^{\mathrm{b}}$ \\ ${ }^{a}$ M.Sc., Computer Science Department, University of Technology, Baghdad, Iraq \\ ${ }^{b}$ Ph.D., Computer Science Department, University of Technology, Baghdad, Iraq
}

\begin{abstract}
In the Past a Few Years, smart devices like smart phones and tablets have radically change in many aspect, started from Entertainment to Shopping services to transfer Money and Banking, the next is Health Services.

With the development in information technology And the big development in cloud computing Here smart phones have entered heavily in all aspects of health care.

Now with the revolution of the smart devices (smart phones or tablets) and it's applications, there is many applications and tools are available started from attachments that allow to diagnose an infections and Now remotely and continuously monitor each heartbeat, blood pressure readings, the rate and depth of breathing, body temperature, oxygen concentration in the blood, glucose, brain waves, activity, mood, so the end result will be can reduce using of doctor, also reduce the cost, and give us speed up and give power to patients, so make it possible for Patient to use portable devices (smart phones or tablet) to access their medical information, and achieve the goal to put information technology to work in health care and make the integration of health information technology into primary care .So the using information technology give us the good solution that won't replace physicians.

Health Information technology give the providers of health care to give better manage patient care. By making the health information are available electronically anytime and anywhere is needed, Health Information technology can help us to improve the quality Of Health, so can decrease the cost.

Now, after all these advantages should shed light on the side of personal privacy And Hacking side that must have been tested all application and tools, All of these tools must be accurate and needs to be tested. So that must provide the highest level of security and privacy for the patients. After that. The application does not arrive the goal with $100 \%$ percent, according to the limitation that mentioned later.
\end{abstract}

Index Terms: Healthcare system, Smart devices application, Iraqi Health care system.

(C) 2017 Published by MECS Publisher. Selection and/or peer review under responsibility of the Research Association of Modern Education and Computer Science.

\footnotetext{
* Corresponding author. Tel.:

E-mail address:
} 


\section{Introduction}

The hospitals and clinic and healthcare environment are different from any other work environments. HC (Health Care System) is very complex because contains different type of Hospitals system like: healthcare providers, dental office, Clinic, insurance, legal issues, patient healthcare providers. Healthcare system will learn and provide the basics of healthcare concepts so can provide the successful on the job and also understand this system.

Now the Healthcare system has become very important thing. Whether in the hospitals, clinic, dental office.

Smart devices (smart phone or tablets) and applications give healthcare providers very good benefits: Improved Accuracy, Enhanced Productivity, better clinical and Decision-Making, better monitoring, Increased Efficiency.

Now there are different applications available now to help Healthcare providers and do important tasks, such as monitoring Diagnose Ear Infections [2], patient record, clinical decision-making, and training.

The smart devices (Smart phones or the tablets) give us very good combination of advantages of computers and communication advantages in the same device, This device can be carried with one hand, provide easy access and easy use in care environment.

Before the development of smart phones, the healthcare providers need good communication to access different types of resources like : email, video, voice, EMRs, EHRs, PACSs , CDSS, so all above resources provided by desktop computers, these computers don't give the portable feature that needed in healthcare.

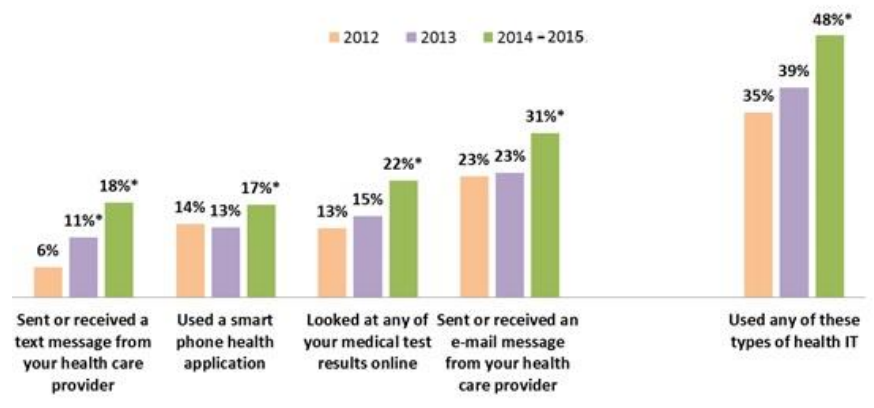

Fig.1.

In Iraq there is some application that help healthcare providers, But this applications are limited possibilities , and built to run on Desktop computers, almost these applications are not doing the analysis or monitoring or other functions for healthcare, these applications often only be booking appointments.

To apply like Healthcare system in Iraq, the environment in Iraq need to:

- $\quad$ stable internet communication

- information security

- $\quad$ awareness

- $\quad$ providing workers and trained personnel

- $\quad$ provides smart phone for patient

\section{Previous Structural Design for Document Health Care System}

For the development of the health system by using modern technologies and take advantage of technological 
progress means to be summarized in a simple, formerly the health system structure before using smart devices techniques and programming sites.

Previous structural health system relies on simple systems and what can hardly be simpler, such as the adoption of traditional medical records or the use of a simplified database is linked in an integrated manner with doctors, nurses and administrators, technicians and workers health system.

In this respect the trend is to introduce the most accurate information techniques to the medical field for use in the health system structural development to become full flexibility to deal with the health system by physicians, patients, administrators, technicians, and maintain the important data in this area (keeping the secret, organize, manage, and making the right decision) and to save time and effort for health providers and thus will meet all the economic, administrative and service purposes in health institutions.

\section{Application Design And Programming for Electronic Healthcare System}

[4][5]In order to provide complete service and using various technology will design and programming mobile and web application for example will use mobile apps to diagnose and remotely monitoring heartbeat, blood pressure readings..etc, and the different between web application and mobile application, the data of web application is stored in server not in mobile device unlike data of mobile application that is stored in mobile device so when use web application not need to install the application because the data is stored in server and other use for web application is best way managing big data.

\section{Implement Native Mobile Application}

In fact, In order to create an application to smart phones or tablets there are top three platforms so in order to create an app for this three platforms, you must understand three operating systems, know how to code in three languages, and understand the UI (User Interface or User Graphic Interface) paradigms of each platform. So to write mobile code, will need to pick a programming language, and in the mobile development world, that language usually matches the dominant language of the platform. So in android the code is Java language, iniOS it's Objective-C or Swift language, and in Windows phones, its C\# or Visual Basic.

Now for the view, will need to define the UI in a platform-specific way. In Android it's defined in an Android XML file, the AXML file in Windows Phone, in the Extensible Application Markup Language, or XAML file, and in iOS use a Xib file or write storyboards. Of course, if prefer to define the UI in code, can do that in each platform, too.

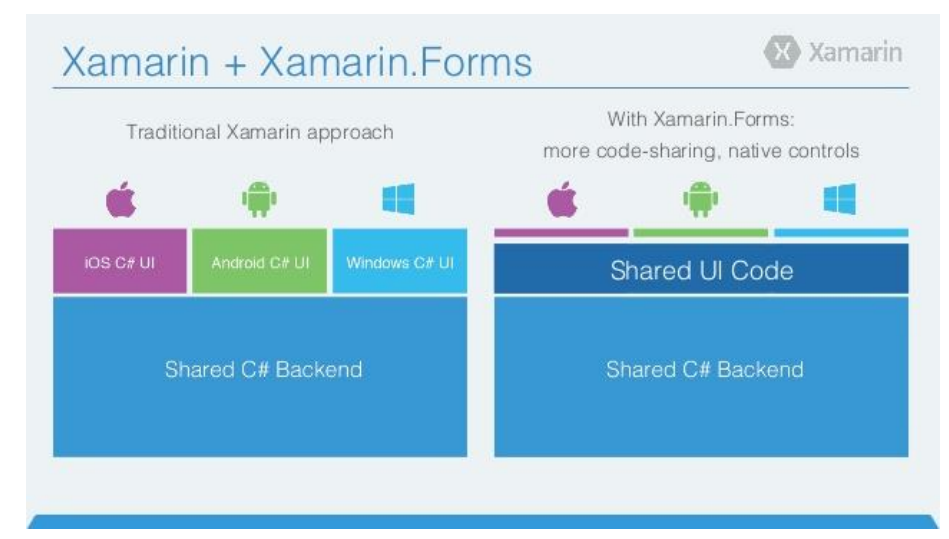

Fig.2. 
So now the Right and perfect choose is [8]Xamarin , because Xamarin seeks to solve this storm and reduce the number of programming languages needed to build apps in all three systems, and it's been successful. It's a popular cross-platform toolkit. Picture below show some shared code native UI.

\section{Native UI From Shared Code}

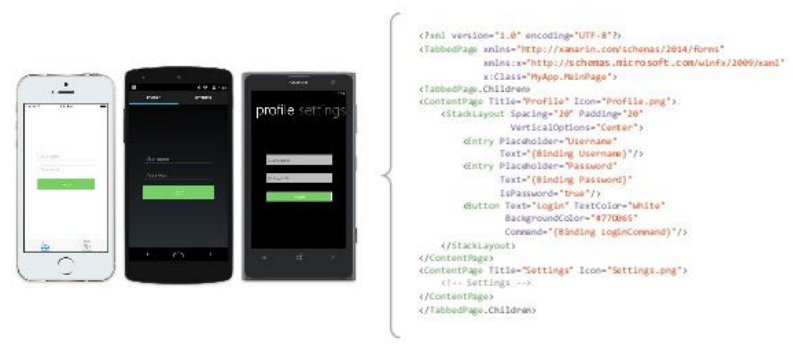

Fig.3.

[6][7]In fact there are various choices available for mobile apps development. But Xamarin aimed directly at the .NET developer. Instead of learning three languages. There are other cross platform mobile tools available, but most of them suffer from performance lags when compared with the apps built with the native languages and tools. Not so with Xamarin. Xamarin apps leverage platform-specific hardware acceleration, and they're compiled for native performance.

The applications that built with xamarin give all features and all functions of native platform. That includes platform capabilities for example: Android Fragments and for iOS iBeacons.

[9]the native Applications can more effectively use phone hardware such as accelerometers, camera and give ability to work and connect to external sensors.

There are three major parts of the Xamarin ecosystem. Xamarin Platform is the main developer part of Xamarin. It provides a set of runtime engines, code APIs, code samples, and virtual machines. The platform targets Android and iOS apps, Mac OS 10 applications, and the Microsoft Windows phones and tablets. Xamarin also has a Test Cloud. It provides an automatic online testing harness that allow application to be tested on thousands of real devices in the Cloud.

The third major part of the system is Xamarin Insights. It is an application monitoring service that tracks app crashes and exceptions and helps the builders know in real time what is happening with applications users.

Now will use Xamarin. Android to build Android apps with Xamarin it is maen work with C\# Language not Java. and define UI (User Interface) and activities in the usual Android way.Xamarin provides the runtime and bindings to make it all work.

And use Xamarin.iOS it is provides the samebenefits, except it's for iOS, and it is also work in C\# Language not Objective-C or Swift. And define UI (User Interface) and views in the usual iOS way.

[10]Xamarin.Forms The key benefit of Xamarin. Forms is that it is truly cross platform. it is mean write shared business code for all the platforms, and define a shared UI with the Microsoft XAML markup language. Without Forms, will need to find three separate UI layers, one for Android, another for iOS, and yet another for Windows devices.

Xamarin is a way to make mobile apps quickly for all platforms with a single, shared C\# code base, build a custom native user interface for each platform, or use Xamarin. Forms to write a single shared user interface across platforms. 


\section{Web Application Implementation}

What is web application: [12] [13] Web applications are application programs that can be accessed over the internet through any device (laptop, mobile, tablet) that have web browser,

[15]MVC is a framework that use to build web applications:

- $\mathrm{M}$ is refers to the Model for dealing with database. $\mathrm{V}$ is refers to the View that is responsible for displaying the data.

- $\mathrm{C}$ is refers to the Controller link between model and view.

- Also by using HTML, CSS, JavaScript can design UI (User Interface). By the end will be able to:

- Create app for top tree platform using Xamarin.[6][8]

- Create an web application using ASP.NET MVC and Web API .[14]

In order to introduce perfect service to patient also need live support so we need to real time functionality so will use SignalR

SignalR[16]: it is a library for ASP.NET developers that simplifies the process of adding real-time web functionality to applications. SignalR can be used to add any sort of "real-time" web functionality to ASP.NET MVC App, for example to build chat application or any real time application.

So this application also can work for Android or IOS or Windows Phone by creating a different UI for those platforms.

\section{Limitations}

- The failure of infrastructure environment for communication and information.

- Network and Information Security.

- The economic situation.

- Lack of awareness.

- Weak communications network.

- The weakness of employee training.

- E-illiteracy.

- The lack of electronic centers close to residential complex.

The table below describe the First 6 months After Appling the healthcare system using smart Devices.

Table 1.

\begin{tabular}{llll}
\hline Month No & Dealing Without HealthCare System & Desktop App & HealthCare System (Smart Devices) \\
\hline 1 & $100 \%$ & $0 \%$ & $0 \%$ \\
2 & $80 \%$ & $20 \%$ & $10 \%$ \\
3 & $70 \%$ & $30 \%$ & $25 \%$ \\
4 & $60 \%$ & $40 \%$ & $40 \%$ \\
5 & $50 \%$ & $45 \%$ & $60 \%$ \\
6 & $30 \%$ & $50 \%$ & $80 \%$ \\
\hline
\end{tabular}




\section{Conclusion}

Healthcare system has many advantages to healthcare providers and patient, Healthcare system can decreases the costs, Improved Accuracy, better monitoring, Increased Efficiency, also give everyone the coverage no matter how sick.

Smart devices (smart phones or tablets) and it's applications are very valuable tools for healthcare providers, and expected to be more widely expanded. But until now some healthcare providers still reluctant to adopt it.

After all the advantages of healthcare system now highly need to make certain the level of quality and safety while using this applications or use this tools . after implement healthcare system expect will be the ability to give very meaningful information and accurate and right in time, also improve the patient results.

\section{References}

[1] Abu Saleh Mohammad Mosa. "Systematic Review of Healthcare Applications for Smartphones"; available at /articles/10.1186/1472-6947-12-67; accessed 2016-02-04.

[2] R.R.Brady, A.C.hunt, M.Arogrigues "Mobile phone technology And hospitalized patients" 2010.

[3] Herbert A. Simon "Transforming Healthcare with Technology. Professor of Computer Science and Robotics Carnegie Mellon University 2016.

[4] Jared Joven "What's the Difference Between a Mobile Website and an App"; Available at accessed 201604-10.

[5] Edward Emmanuel Which is better: "A native app or web app"; available at accessed 2016-.,search engine optimization specialist.

[6] Hermes, Dan "Understanding the Xamarin Mobile Platform" 2016.

[7] Hermes, Dan "What is XAMARIN? Is it a framework? Or compiler? Or is it a just a code translator?" 2016.

[8] Telerik, academy school "Introduction to Xamarin" 2015.

[9] Charalam lampos Doukas "Mobile healthcare information management" 2014.

[10] James Montemagno, "Xamarin.Forms - Write Once, Run Everywhere;getting started" 2015.

[11] Rahul Sahay "ASP.NET MVC frameworkIntroduction" 2015

[12] Md. Shahadat Hussain "Medical healthcare web application" 2016.

[13] Masaaki Kurosu, "native apps.versus web app.wich the best for healthcare, 2013.

[14] Elizer Geisler, Ori Heller, "management of medical technology, 2013

[15] Monsey, NY, us "ASP.net software developer-see connections who works in healthcare, 2015.

[16] Dan Wahlin, "ASP.net healthcare toolkit controls" 2016.

\section{Authors' Profiles}

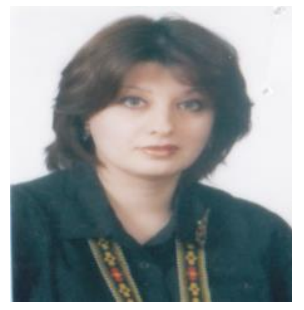

Suhiar M.Zeki was born in Baghdad, Iraq, she obtained B.SC. (1994), MSC (2005) in computer science from Iraqi commission for computers informatics, informatics institute for postgraduate studies, she teaches in computer science department / University of technology /Iraq/Baghdad, and currently she is a PH.D student at the technology university /Baghdad, her research interests are: healthcare systems, data warehouse, data management, data security , artificial intelligent.

She has a certificate for practitioner (self-developing messages project) from creativity horizons for training, human development \&administrative improvement (chthai)

/Iraq/2014. 
Prof. Abdul Monem S. Rahma Ph.D Awarded his M.Sc. from Brunel University and his $\mathrm{Ph} . \mathrm{D}$. from Loughborough University of technology United Kingdom in 1982, 1984 respectively. He taught at Baghdad university Department of Computer Science and the Military Collage of Engineering, Computer Engineering Department from 1986 till 2003.

From 2004 to 2012 he holds the position of Dean Asst. and from 2013 to 2015 he holds the position of Dean of the Computer Science Department at the University of Technology.

He published 130 Papers, 4 Books in the field of computer science, supervised $31 \mathrm{Ph} . \mathrm{D}$. and 63 M.Sc. students.

His research interests include Computer Graphics Image Processing, Biometrics and Computer Security.

How to cite this paper: Suhiar Mohammed Zeki, Abdul Monem Saleh Rahma,"Healthcare System Technology using Smart Phones and Web Apps (Case Study Iraqi Environment)", International Journal of Engineering and Manufacturing(IJEM), Vol.7, No.3, pp.1-7, 2017.DOI: 10.5815/ijem.2017.03.01 